\title{
Gender-Dimorphic Regulation of Skeletal Muscle Proteins in Streptozotocin-Induced Diabetic Rats
}

\author{
Minji Choi Jung-Won Choi Harmesh N. Chaudhari Kanikkai Raja Aseer Rajib Mukherjee
}

Jong Won Yun

Department of Biotechnology, Daegu University, Kyungsan, Kyungbuk 712-714, Republic of Korea

\section{Key Words}

2-DE $\cdot$ Diabetes $\bullet$ Gender differences $・$ Muscle proteome $\cdot$ Streptozotocin

\begin{abstract}
Background: Despite the fact that sexual differences increase diabetic risk and contribute to the need for gender-specific care, there remain contradictory results as to whether or not sexual dimorphism increases susceptibility to the development of type 1 diabetes mellitus. Methods: To examine gender-dimorphic regulation of skeletal muscle proteins between healthy control and STZ-induced diabetic rats of both genders, we performed differential proteome analysis using two-dimensional electrophoresis combined with mass spectrometry. Results: Animal experiments revealed that STZ treatment rendered female rats more susceptible to induction of diabetes than their male littermates with significantly lower plasma insulin levels due to hormonal regulation. Proteomic analysis of skeletal muscle identified a total of 21 proteins showing gender-dimorphic differential expression patterns between healthy controls and diabetic rats. Most interestingly, gender-specific proteome comparison showed that male and female rats displayed differential regulation of proteins involved in muscle contraction, carbohydrate, and lipid metabolism, as well as oxidative phosphorylation and cellular stress. Conclusion: The current proteomic study revealed that impaired protein regulation was more prominent in the muscle tissue of female diabetic rats, which were more susceptible to STZinduced diabetes. We expect that the present proteomic data can provide valuable information for evidence-based gender-specific treatment of diabetes.
\end{abstract}

Copyright $(2013$ S. Karger AG, Basel

\section{Introduction}

Gender differences in physiology and metabolism have important potential implications with regards to the development of new approaches for the intervention of metabolic diseases such as diabetes and obesity [1]. A growing body of evidence indicates that sexual differences increase diabetic risk and contribute to the need for gender-specific care [25]. However, to date, no proteomic studies have investigated this relationship as a primary outcome. 
Type 1 diabetes mellitus (T1DM) results from autoimmune destruction of pancreatic $\beta$-cells, leading to absolute insulin deficiency in patients as well as a requirement for exogenous insulin supplementation for survival [6]. To induce experimental T1DM in animals, streptozotocin (STZ) has been widely used due to its cytotoxic activity against pancreatic $\beta$-cells $[7,8]$, and chronic STZ-induced diabetic rodents mimic diabetic complications observed in diabetic humans [9].

There is evidence of sexual dimorphism in the incidence and prevalence of diabetes in T1DM animal models, but relevant results are still not definitive. For example, it was demonstrated that male mice are more vulnerable to STZ and thus exhibit higher loss of $\beta$-cells, whereas female rodents show lower prevalence of T1DM due to the protective effect of estrogen on $\beta$-cell apoptosis $[10,11]$. Conversely, female animal models show higher blood glucose levels than their age-matched male littermates when treated with STZ due to greater disorganization of islets and insulin insensitivity $[12,13]$. There is now a consensus that sexual-dimorphic prevalence of T1DM is strongly influenced by sex hormones, particularly estrogen $[14,15]$. However, it is not simply a matter of sex hormones as the underlying mechanism may be more complex. Thus, to more definitely conclude whether males or females show predominance of T1DM, more systemic studies are required.

Skeletal muscle is the major site of metabolic fuel utilization in the body as well as an organ for insulin-mediated glucose disposal. Thus, cellular dysfunctions of muscle are one of the most important pathophysiological features of diabetes [16]. To date, several previous biochemical studies have investigated gender-specific dimorphism in the regulation of skeletal muscle proteins in terms of differences in insulin resistance [17], strength and fiber distribution $[18,19]$, mitochondrial function [20], oxidative metabolism [21] and fatigability [22] in mammals. However, to the best of our knowledge, no studies have attempted to characterize gender-dependent alterations of diabetic skeletal muscle at the proteomic level.

In the present study, we compared differentially regulated proteins in skeletal muscle between healthy control and STZ-induced rats of both genders by 2-DE-based proteomic analysis. To the best of our knowledge, this is the first proteomic approach to address gender-specific dimorphism in diabetic animals, particularly in STZ-induced diabetic rats.

\section{Materials and Methods}

\section{Animal experiments}

Eleven-week-old Sprague-Dawley (SD) male and female rats were purchased from Daehan Experimental Animals (Seoul, Korea) and kept at one rat per cage at $24 \pm 2{ }^{\circ} \mathrm{C}$. After 1 week of adaptation, 24 male and 24 female rats were randomly divided into two groups. The control group contained eight male and eight female rats, whereas the STZ-treated group consisted of 16 males and 16 females. All groups were supplied a normal diet (12\% fat calories). To induce diabetes, STZ dissolved in $0.01 \mathrm{M}$ sodium citrate buffer ( $\mathrm{pH} 4.5$ ) was injected intravenously into the STZ-treated group of males and females at a dose of $50 \mathrm{mg} / \mathrm{kg}$ body weight, whereas control rats were injected with vehicle alone. Glucose levels and body weight were measured at the same time point over a period of 3 weeks. Fasting blood glucose levels were determined using glucose oxidase reagent strips (Lifescan, Milpitas, CA, USA), and rats with a blood glucose level $>300$ $\mathrm{mg} / \mathrm{dL}(16.7 \mathrm{mmol} / \mathrm{L})$ were judged as diabetic. Finally, we selected six rats from each group. After 2 weeks, all animals were fasted for a period of $12 \mathrm{~h}$, after which skeletal muscle tissue samples were collected. Experiments were approved by the Committee for Laboratory Animal Care and Use of Daegu University. All procedures were conducted in accordance with the Guide for the Care and Use of Laboratory Animals published by the National Institutes of Health.

\section{Biochemical parameters of plasma}

Blood samples were obtained from the tails of rats under anesthesia and were collected in EDTA tubes. Plasma was separated by centrifugation $(3,000 \times g, 10 \mathrm{~min})$ and kept at $-80^{\circ} \mathrm{C}$. Plasma insulin, leptin, and sex hormones were measured using a sandwich enzyme-linked immunosorbent assay (ELISA) system, including a Rat Insulin kit (ALPCO, Salem, NH, USA), Rat Leptin kit (Enzo Life Sciences, Farmingdale, NY, 
Choi et al.: Gender Dimorphism in Skeletal Muscle Proteome

USA), Rat 17- $\beta$-Estradiol high sensitivity ELISA kit (Enzo Life Sciences), and Rat Testosterone ELISA kit (Enzo Life Sciences). Each assay was performed in triplicate using individual plasma samples of six selected rats per group according to the manufacturer's protocol.

Preparation of skeletal muscle samples for two-dimensional electrophoresis (2-DE)

Soleus skeletal muscles were excised from hindlimb of the anesthetized rats. Excised muscle tissues were washed in cold saline solution and then crushed in liquid nitrogen. Samples were homogenized in rehydration buffer consisting of 7 M Urea (Bio Basic, Ontario, Canada), 2 M thiourea (Sigma, St. Louis, MO, USA), 4\% CHAPS (Bio Basic), 1 mM PMSF (Sigma), 20 mM DTT (GE Healthcare, Buckinghamshire, UK), and 2\% IPG buffer (Bio-Rad, Hercules, CA, USA) using a homogenizer (PT 1200E, Kinematica Ltd., Luzern, Switzerland) on ice. Extracts were centrifuged at 13,000 $\times \mathrm{g}$ for $20 \mathrm{~min}$, after which the supernatant was stored at $-80^{\circ} \mathrm{C}$ until analysis. Protein concentration in the muscle tissues was measured by the Bradford method [23] using protein assay dye reagent concentrate (Bio-Rad).

\section{2-DE analysis}

2-DE was performed using protein extracts from the muscles of six rats per group, as described previously [24, 25]. For minimization of gel-to-gel variation, this experiment pooled muscle protein extracts from six rats, and three sheets of 2-DE gels per group were prepared. IPG strips of pH 4-7 non-linear (18 $\mathrm{cm}$; GE Healthcare) were rehydrated for $20 \mathrm{~h}$ in rehydration buffer containing $100 \mu \mathrm{g}$ of protein. Isoelectric focusing (IEF) was conducted using the PROTEIN IEF cell (Bio-Rad, Hercules, CA, USA) at $20^{\circ} \mathrm{C}, 15 \mathrm{~min}$ at $250 \mathrm{~V}, 3 \mathrm{~h}$ at 250-10,000 V, $6 \mathrm{~h}$ at 10,000 V, and then held at $500 \mathrm{~V}$. For the second dimension, gel strips were equilibrated for 15 min in 6 M urea, 2\% SDS (Generay Biotech, Shanghai, China), 30\% glycerol (Bio Basic), $50 \mathrm{mM}$ Tris (pH 8.8), and 1\% DTT, followed by replacement of DTT with 2.5\% iodoacetamide (Bio-Rad) for $15 \mathrm{~min}$. Gel strips were then subjected to $20 \times 20 \mathrm{~cm}^{2} 10 \%$ SDS-polyacrylamide gel electrophoresis, after which fractionation was performed on the Laemmli SDS-discontinuous system at a constant voltage of 15 mA per gel. The separated gels were visualized by silver staining as described previously [24, 25].

\section{Image acquisition and data analysis}

Proteins spots in gels imaged by a UMAX PowerLook 1120 (Maxium Technologies, Akron, OH, USA) were compared between groups using modified ImageMaster 2-D software V4.95 (GE Healthcare). A reference gel was selected from gels of the control group, and detected spots were matched with those in the reference gel. Relative optical density and relative volume were calculated for correction of differences in gel staining. Intensity volume of individual spots was processed by background subtraction and total spot volume normalization; the resulting spot volume percentage (\% volume) was used for comparison as described previously [24].

\section{Peptide mass fingerprinting (PMF)}

All chemicals used in this study were analytical grade and purchased from Sigma. Protein spots were enzymatically-digested in-gel in a manner similar to that previously described by Shevchenko et al. [26] using modified porcine trypsin (Promega, Madison, WI, USA). Gel pieces were washed with 50\% acetonitrile to remove SDS, salt, and stain. Washed and dehydrated spots were then vacuum-dried to remove solvent, rehydrated with trypsin (8-10 $\mathrm{ng} / \mu \mathrm{L})$ solution in $50 \mathrm{mM}$ ammonium bicarbonate (pH 8.7), and incubated $8-10 \mathrm{~h}$ at $37^{\circ} \mathrm{C}$. Samples were analyzed using an Applied Biosystems 4700 proteomics analyzer (Foster City, CA, USA) with TOF/TOF ${ }^{\mathrm{TM}}$ ion optics. Both MS and MS/MS data were acquired with a Nd:YAG laser at a repetition rate of $200 \mathrm{~Hz}$, and up to 4000 shots were accumulated for each spectrum. MS/MS mode was operated at a collision energy of $2 \mathrm{keV}$; air was used as the collision gas such that nominally single collision conditions were achieved. Although the precursor selection has a possible resolution of 200, a resolution of 100 was utilized in these studies on known single component analytes. Both MS and MS/MS data were acquired using the instrument default calibration without applying any internal or external calibration. Sequence tag searches were performed with the program MASCOT (http://www.matrixscience. com).

Immunoblot analysis

In this study, the levels of six proteins identified on a 2-DE map of rat muscle were verified by immunoblot analysis. Tissue lysates were prepared with RIPA buffer (Sigma), homogenized, and centrifuged 

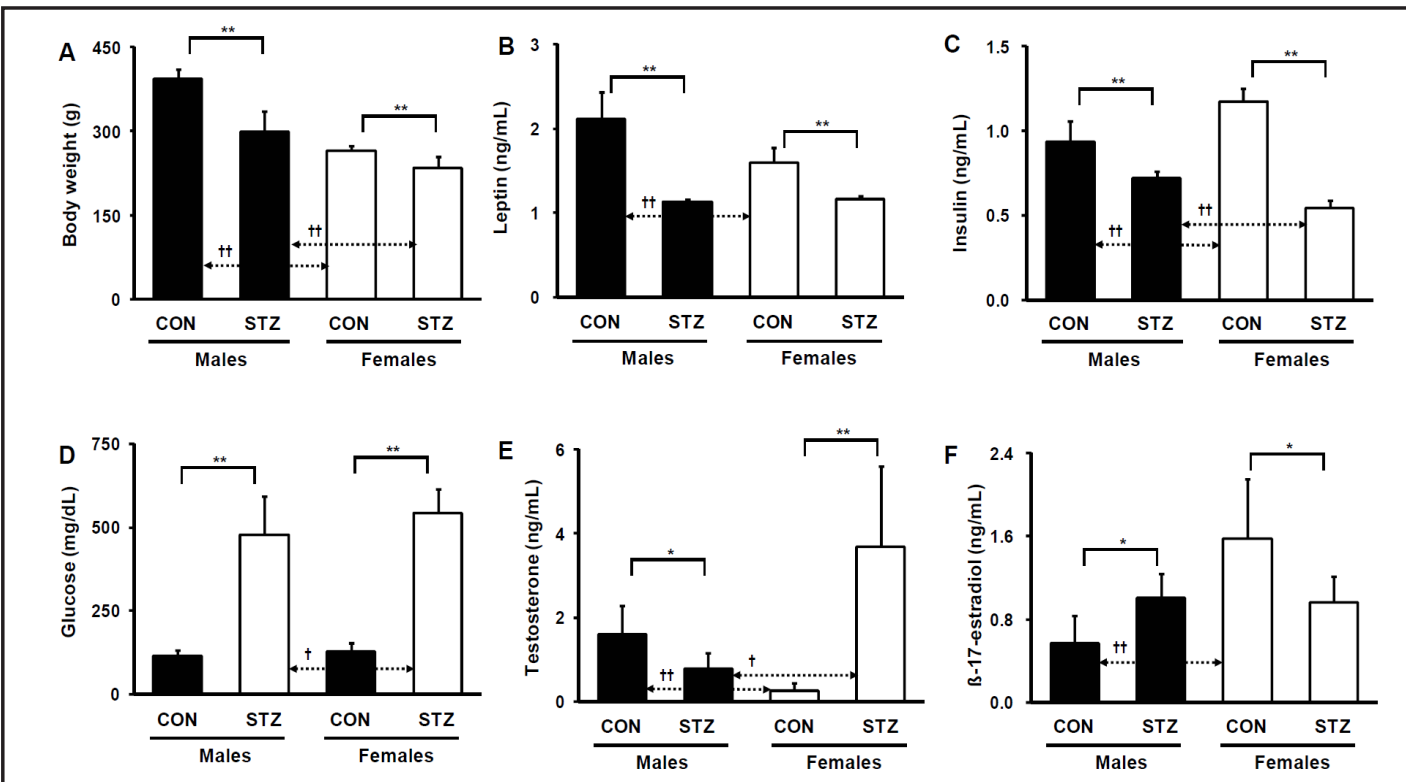

Fig. 1. Alterations of body weights (A) as well as plasma levels of leptin (B), insulin (C), glucose (D), testosterone (E), and $\beta$-17-estradiol (F) in control and STZ-induced diabetic male and female rats after 2 weeks of STZ treatment. Data are presented as the mean \pm SD in the group and were estimated by ANOVA test. Statistical significance between control and STZ-treated rats was determined by a Student's $t$-test, where $p$ value is ${ }^{*} p<0.05$ and ${ }^{* *} p<0.01$. Significance between male and female rats was represented by ${ }^{\dagger} p<0.05$ and ${ }^{\dagger \dagger} p<0.01$.

at $12,000 \times g$ for $20 \mathrm{~min}$. Extract was diluted in $5 \mathrm{X}$ sample buffer (50 mM Tris (pH 6.8), 2\% SDS, 10\% glycerol, $5 \% \beta$-mercaptoethanol, and $0.1 \%$ bromophenol blue) and heated for $5 \mathrm{~min}$ at $95^{\circ} \mathrm{C}$. After 6,8 , 10 , or $12 \%$ SDS-polyacrylamide gel electrophoresis (PAGE), samples were transferred to a polyvinylidene difluoride membrane (Santa Cruz Biotechnology, Santa Cruz, CA, USA) and blocked for $1 \mathrm{~h}$ with TBS (Trisbuffered saline)-T buffer (10 mM Tris- $\mathrm{HCl}, 150 \mathrm{mM} \mathrm{NaCl}, 0.1 \%$ Tween 20 containing 5\% skim milk). The transferred membrane was incubated for $1 \mathrm{~h}$ with a 1:1000 dilution of primary polyclonal anti-Atp5B, anti-GP, anti-PGM-1 (Santa Cruz Biotechnology), anti-TPM1 (Abcam, Cambridge, UK), anti-Eno1, and antiEno3 (Proteintech, Chicago, IL, USA) antibody in TBS-T buffer containing 1\% skim milk after washing using TBS-T buffer. After washing, the membrane was incubated for $1 \mathrm{~h}$ with horseradish peroxidase-conjugated anti-goat IgG, anti-mouse IgG, or anti-rabbit IgG secondary antibody (1:1000, AB Frontier, Seoul, Korea) in TBS-T buffer containing 1\% skim milk and developed using enhanced chemiluminescence (GE Healthcare). Immunoblot analysis was performed by scanning with a UMAX PowerLook 1120 and digitalized using image analysis software (KODAK 1D, Eastman Kodak, Rochester, NY, USA).

\section{Statistical analysis}

All experimental results were compared by one-way analysis of variance (ANOVA) using the Statistical Package of Social Science (SPSS) program, and data were expressed as the mean \pm SD. Group means were considered significantly different at $p<0.05$, as determined by the technique of protective least-significant difference (LSD) when ANOVA indicated an overall significant treatment effect.

\section{Results}

Differences in diabetic phenotype between male and female rats in response to STZ treatment

Prior to the proteomics study, we first conducted an animal experiment in order to examine alterations of body weight as well as plasma levels of blood glucose, insulin, leptin, and sex hormones between healthy control and STZ-induced diabetic rats of both 
Fig. 2. Representative silver-stained 2-DE image of rat skeletal muscle proteome for each experimental group. Twenty proteins showing differential regulation in a genderdependent manner are marked with their abbreviated names (see the gel for Male-CON). Full names of proteins are presented in the abbreviations section.
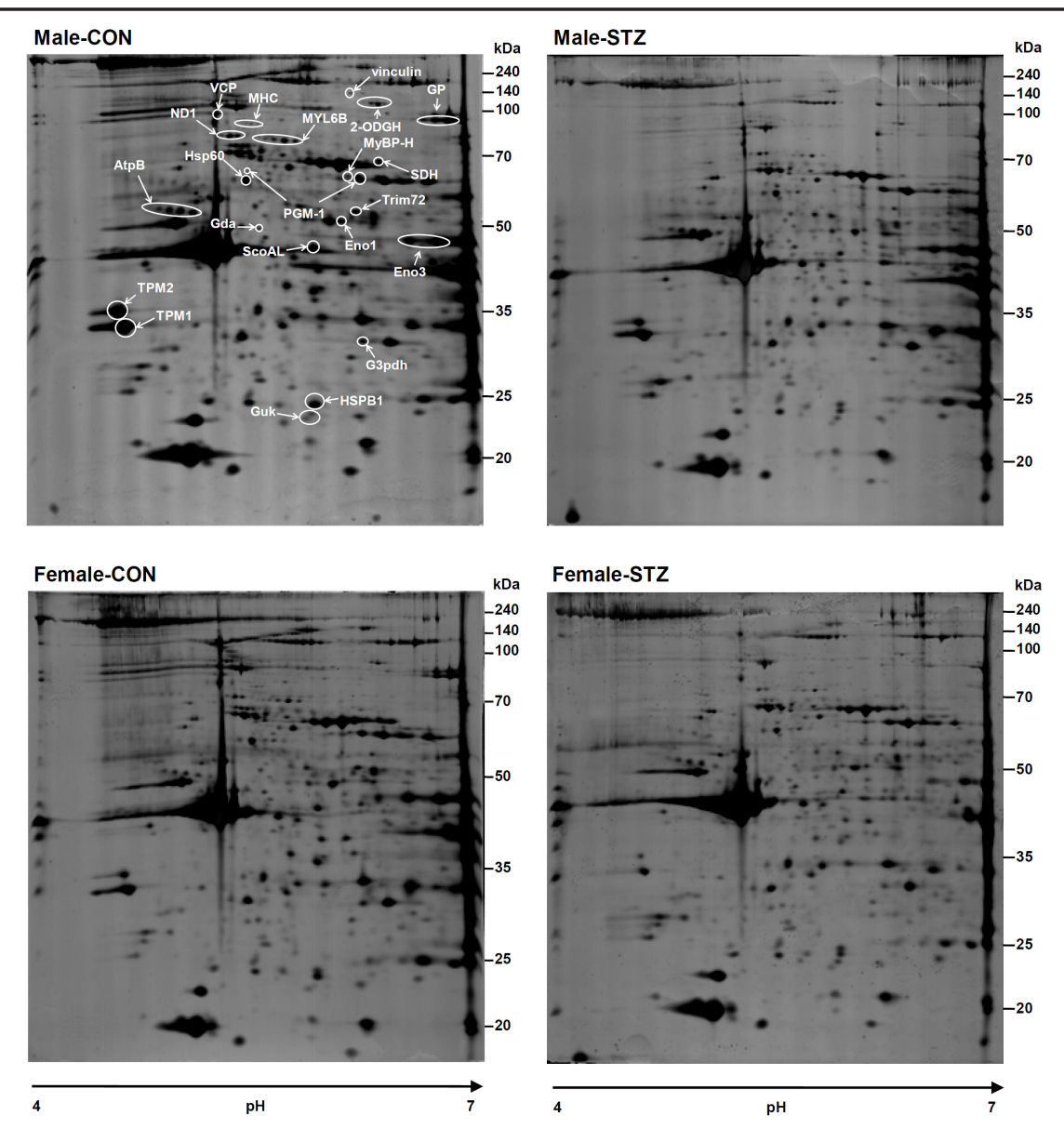

genders. Two weeks after STZ treatment, male rats showed greater body weight reduction than female rats compared to their control littermates (Fig. 1A), which was in agreement with higher reduction of plasma leptin levels due to loss of fat in diabetic male rats (Fig. 1B). Conversely, significantly lower plasma insulin levels were observed in female diabetic rats (Fig. 1C), resulting in higher blood glucose levels (Fig. 1D). Importantly, we measured significantly different plasma sex hormone levels between male and female rats. Expectedly, plasma testosterone levels were markedly reduced upon STZ treatment in male rats, whereas levels were remarkably elevated in female diabetic rats (Fig. 1E). In contrast, plasma levels of estrogen ( $\beta$-17-estradiol) were higher in males but reduced in females upon treatment with STZ (Fig. 1F). Greater reduction of plasma insulin levels in female rats was closely associated with reduced levels of estrogen, which is a potent stimulator of insulin secretion in pancreatic islets.

\section{Proteome analysis}

Proteins were resolved by 2-DE using two different gel systems. First, proteins were resolved on a broad-range IEF strip ( $\mathrm{pH} 3-10$, non-linear gradient) in the first dimension and on a $12 \%$ SDS-PAGE gel in the second dimension, as outlined by our previous studies [24,27]. However, improved resolution could be obtained when the first dimension separation was carried out on a pH 4-7 rather than pH 3-10 IEF strip. Therefore, we chose pH 4-7 gels for the remainder of the study in order to take advantage of their greater resolution. As shown in Fig. 2 , over 650 individual spots were detected, ranging from 6 to $200 \mathrm{kDa}$ in mass between $\mathrm{pH}$ 4-7. Among them, we identified a total of 21 proteins showing sexually dimorphic regulation patterns between both healthy control as well as diabetic male and female rats (Table 1). 

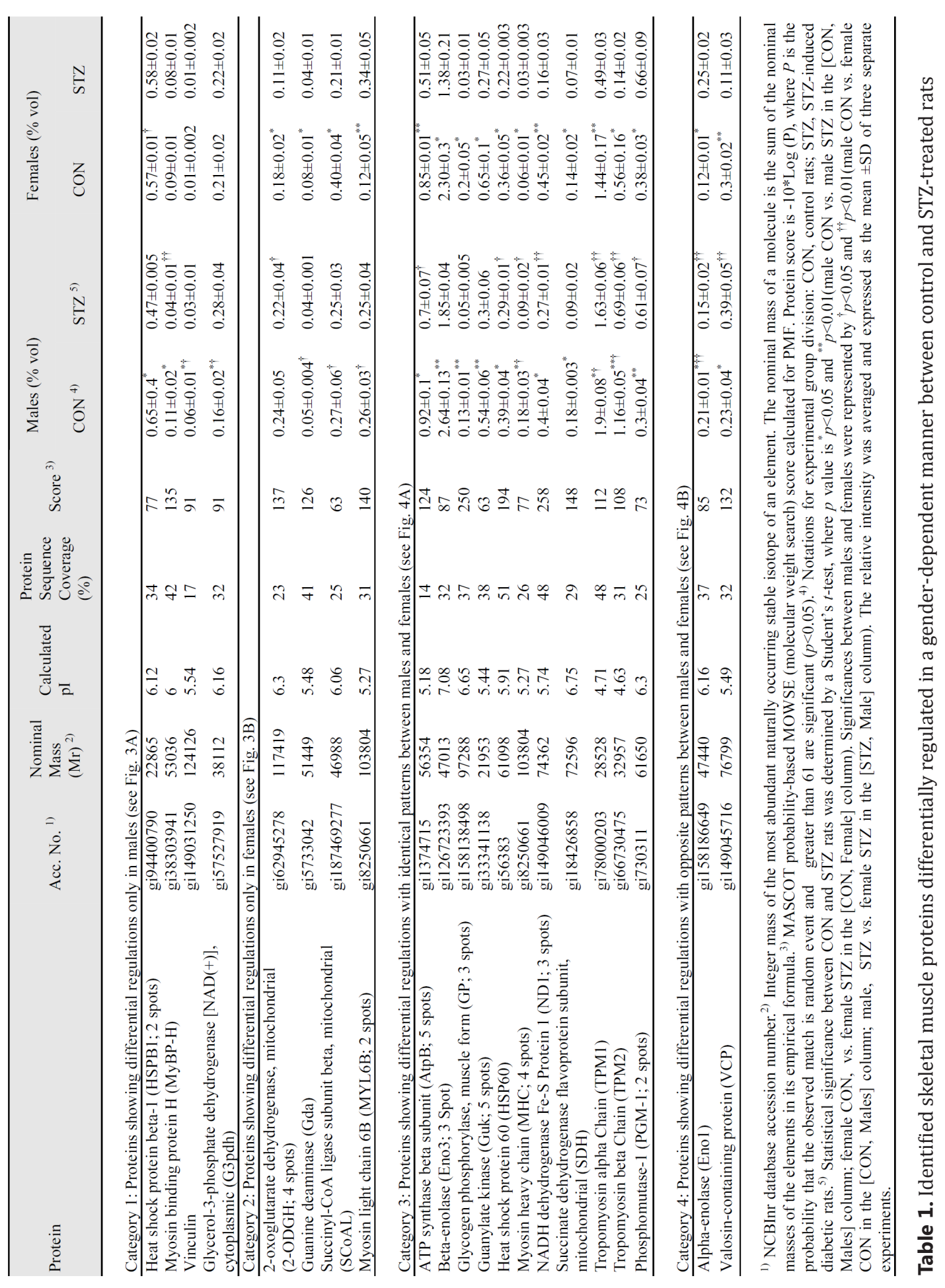

Gender-dimorphic regulation of skeletal muscle proteins

To compare protein regulation among the groups, we categorized the identified proteins into four groups by analyzing pooled muscle samples from six rats per group. Category (1) includes proteins showing differential regulation only in males (healthy male control vs. diabetic male rats). Four proteins were included in this category, namely HSPB1, MyBP-H, 


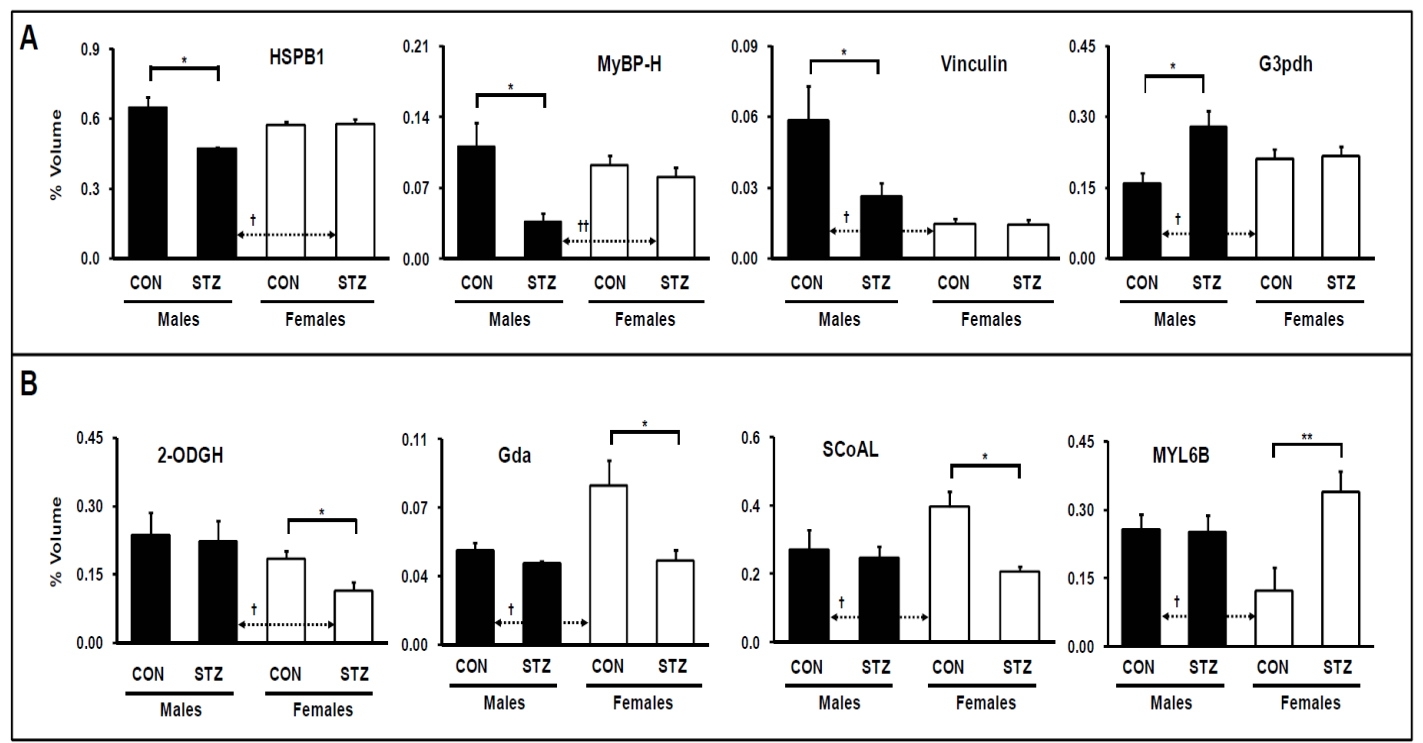

Fig. 3. Skeletal muscle proteins showing differential regulation only in males (healthy male control vs. diabetic male rats; panel A) or in females (healthy female control vs. diabetic female rats; panel B). Data are presented as the mean \pm SD of volume density (\%) of the changed protein in pooled samples from six rats per group. Statistical significance between control and STZ-treated rats was determined by ANOVA test, where $p$ value is ${ }^{*} p<0.05$ and ${ }^{* *} p<0.01$. Significance between male and female rats was represented by ${ }^{\dagger} p<0.05$ and ${ }^{\dagger \dagger} p<0.01$. Full names of proteins are presented in the abbreviations section.

vinculin, and G3pdh (Fig. 3A). Category (2) includes proteins showing differential regulation only in females (healthy female control vs. diabetic female rats). Four proteins were included in this category, namely 2-ODGH, Gda, SCoAL, and MYL6B (Fig. 3B). Category (3) includes proteins showing identical patterns of differential regulation between both healthy control as well as diabetic male and female rats (healthy male control vs. diabetic male rats; healthy female control vs. diabetic female rats). Eleven proteins were included in this category, namely AtpB, Eno3, GP, Guk, HSP60, MHC, ND1, SDH, TPM1, TPM2, and PGM-1 (Fig. 4A). Category (4) includes proteins showing inverse patterns of differential regulation between both control as well as diabetic male and female rats (healthy male control vs. diabetic male rats; healthy female control vs. diabetic female rats). Two proteins were included in this category, namely Eno1 and VCP (Fig. 4B).

\section{Validation of proteomic data using Western blot analysis}

Although a total of 21 proteins showed significantly differential regulation in a genderdependent manner, we were unable to exclude the possibility of technical errors or artificial effects in our proteomic analysis. To clarify this, the plasma protein levels of six proteins of interest were further investigated by immunoblot analysis. As shown in Fig. 5, we confirmed that the regulation patterns of all examined proteins were exactly in line with those of the Western blot analysis.

\section{Discussion}

The results of the current animal experiment revealed that female rats were more susceptible to diabetes onset upon STZ treatment, which raises the question of what changes contribute to this sexual dimorphism. We postulated that abnormally increased levels of testosterone along with significantly reduced levels of estrogen in female diabetic rats, at least in part, contributed to greater dysfunction of skeletal muscle compared to their male 


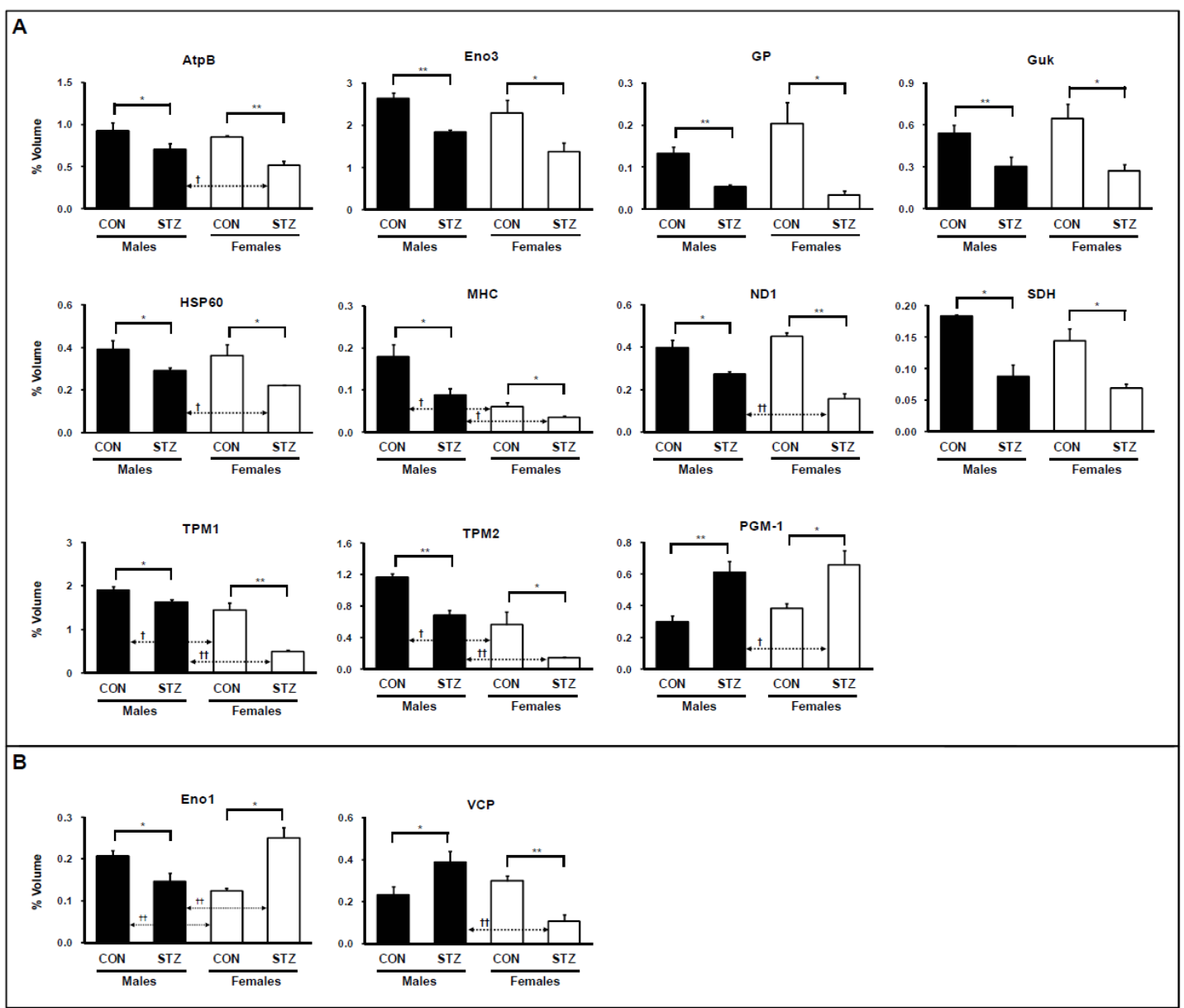

Fig. 4. Skeletal muscle proteins showing identical (panel A) or inverse patterns (panel B) of differential regulation between both males and females (healthy male control vs. diabetic male rats; healthy female control vs. diabetic female rats). Data are presented as the mean \pm SD of volume density (\%) of the changed proteins in pooled samples from six rats per group. Statistical significance between control and STZtreated rats was determined by ANOVA test, where $p$ value is ${ }^{*} p<0.05$ and ${ }^{* *} p<0.01$. Significance between male and female rats was represented by ${ }^{\dagger} p<0.05$ and ${ }^{\dagger+} p<0.01$. Full names of proteins are presented in the abbreviations section.

counterparts. A previous study also reported that estrogen may play a protective role against the effects of glucose toxicity in skeletal muscle and improves insulin sensitivity, whereas testosterone is unable to mediate its protective effects against chronic hyperglycemia and reduce insulin sensitivity [28]. Taken together, impaired biosynthesis and reduced plasma concentration of estrogen were sensitive events upon STZ treatment, thereby increasing susceptibility to diabetes incidence in females. Based on the possibility that hormonal changes may affect gender-dependent protein regulation in the majority of metabolic tissues, we attempted to determine how STZ treatment caused proteomic alterations in skeletal muscle, which is the major site of metabolic fuel utilization in the body.

We successfully identified numerous proteins showing gender-specific dimorphism in skeletal muscle between healthy control and STZ-induced diabetic rats. Most interesting is that our gender-specific proteome comparison showed that male and female rats displayed different regulation patterns of proteins involved in muscle contraction, carbohydrate and lipid metabolism, as well as oxidative phosphorylation and cellular stress.

One of the interesting results in this proteomic study is the down-regulation of ATP synthase beta subunits (AtpB) in the muscle tissues of both male and female diabetic rats. 
Fig. 5. Validation of skeletal muscle proteins showing differential regulation between healthy control and diabetic rats by immunoblot analysis. Levels of six identified proteins from 2-DE analysis were established using a pooled sample from six rats per group, and data are representative of three independent experiments. Band density was calculated using ImageMaster 2D software version 4.95, and relative intensity (\%) values of proteins were normalized to $\beta$-actin levels. Statistical significance between control and STZtreated rats was determined by ANOVA test, where $p$ value is ${ }^{*} p<0.05$. Significance between male and female rats was represented by ${ }^{\dagger} p<0.05$. Full names of proteins are presented in the abbreviations section.
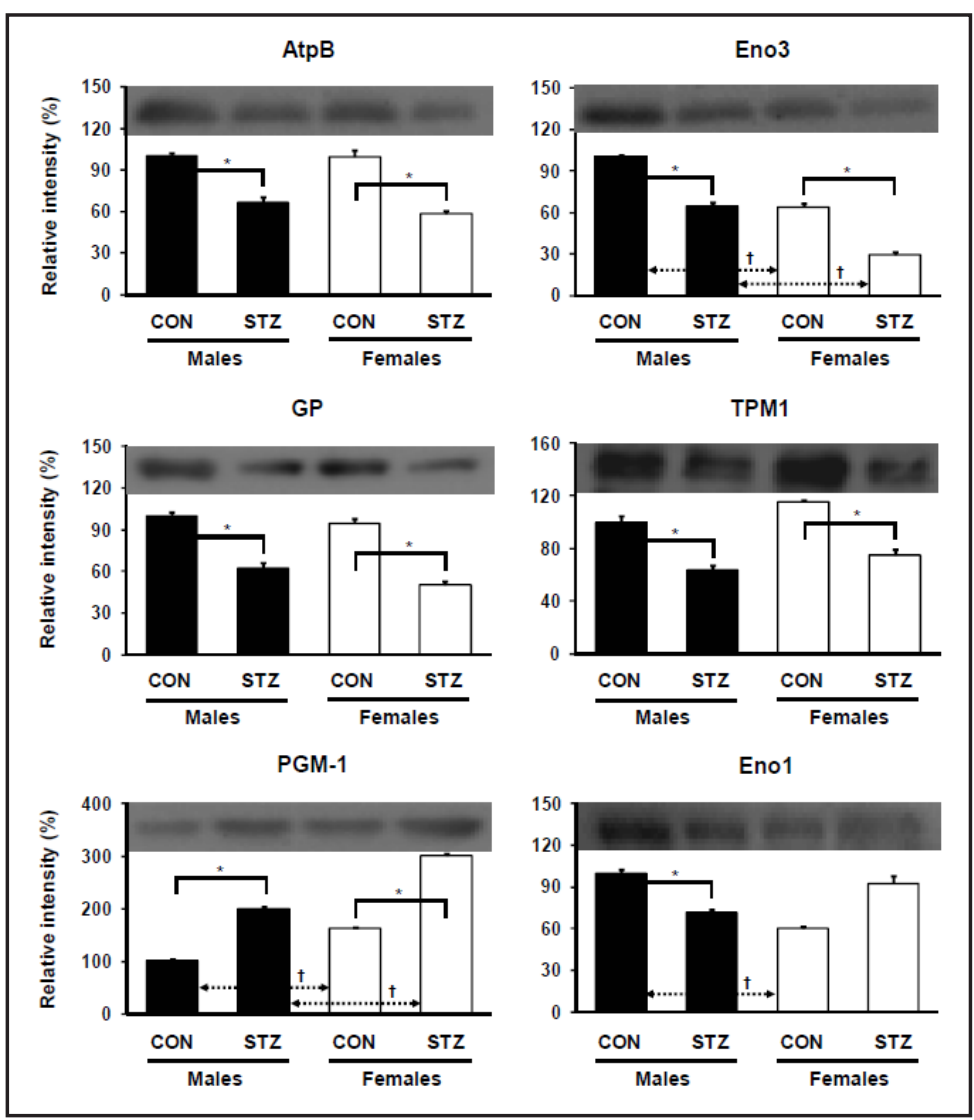

Greater reduction of AtpB protein levels in skeletal muscle was closely associated with greater reduction of insulin levels in female diabetic rats (Fig. 1C). An earlier report revealed down-regulation of AtpB in the skeletal muscle of patients with T2DM [29]. Collectively, reduced expression of AtpB may contribute to the pathogenesis of both T1DM and T2DM.

We also detected reduced expression of NADH dehydrogenase subunit 1(ND) in diabetic rats of both genders, with greater reduction in females compared to healthy controls, similar to that observed in T2DM subjects [30, 31]. Impaired expression of ND1 represents mitochondrial metabolic derangement during the development of diabetes [30]. Taken together with the results of reduced AtpB expression, STZ-induced diabetic rats showed disrupted mitochondrial bioenergetic functions due to lower expression of electron transport proteins, with greater dysfunction in females. This reduced expression of electron transport proteins and energy production led to deceleration of the TCA cycle, as evidenced by reduced expression of three TCA cycle enzymes (e.g. 2-ODGH, SDH, and SCoAL) in this proteomic study [32, 33].

Interestingly, we for the first time observed gender-dimorphic expression of several contractile muscle proteins. The first example is reduced expression of myosin heavy chain (MHC), which is associated with the speed of muscular contraction. In vitro experiments have demonstrated that insulin stimulates synthesis of MHC [34]. However, it was also demonstrated that short-term insulin deprivation has no effect on the synthesis of MHC [35]. Similarly, we also detected reduced expression of two forms of tropomyosins (TPM1 and TPM2), which play a key role in the structure and regulation of muscle contractions [36]. Although mutation in the TPM2-encoding gene has recently been found to cause a range of muscle disorders [37], this study is the first to observe reduced expression of these proteins in T1DM animal models. Likewise, myosin-binding protein $\mathrm{H}(\mathrm{MyBP}-\mathrm{H})$ was also significantly down-regulated only in male and not in female diabetic rats. Greater reduction of MyBP protein levels in male diabetic rats was not simply a result of glycation as higher blood 
glucose levels were observed in female rats (Fig. 1B). Since MyBP is known to be associated with assembly of myofibrils containing myofilaments of appropriate length and composition for effective contractile function [38], down-regulation of MyBP reflects impairment of muscle function and diabetes-related contractile weakness. Taken together, insulin-deficient diabetes elicits alterations of contractile protein synthesis as well as marked changes in muscle function, which may be hallmarks of diabetic muscle as observed in diabetic heart tissue [39]. However, we unexpectedly observed significant elevation of myosin light chain 6B (MYL6B) expression in diabetic females, whereas no alterations were observed in males upon STZ treatment (Fig. 3). The mechanism underlying gender differences in the expression of this protein is unclear, and thus further studies are warranted to examine in detail the physiological significance of this difference.

Clear gender dimorphism in vinculin regulation was also observed, in that significantly higher expression was detected in normal healthy males than their female counterparts. Moreover, reduced expression of vinculin was observed in male but not female diabetic rat muscle, which was comparable with previous observations that vinculin is down-regulated in bladder smooth muscle tissue of STZ-induced diabetic rats [40]. Considering its regulatory function in skeletal muscle, reduced expression of vinculin in diabetic rats reflects a modified mechanical link between the actin cytoskeleton and integrin [40]. However, the result that vinculin regulation was not altered in female rat muscle before and after STZ treatment could not be interpreted. Further studies are likely to yield important information about the precise role of vinculin in the pathophysiology of diabetes.

Another interesting finding in the current proteomic study is that diabetic rats of both genders showed reduced expression of guanylate kinase (ATP:(d)GMP phosphotransferase, EC 2.7.4.8), which catalyzes the ATP-dependent phosphorylation of GMP into GDP [41]. An earlier report demonstrated that insulin exerts a profound effect on liver purine metabolism by regulating nucleotide concentrations [42]. We concluded that STZ-treatment decreased cellular concentrations of ATP in rat muscle tissue, thereby impairing purine metabolism in diabetic rats.

We also detected reduced expression of two heat shock proteins (HPS60 and HSPB1) in diabetic male rats only, suggesting impaired activation of the stress response as well as atrophy of certain muscles or muscle fibers during development of diabetes. Our proteomic data support earlier findings that constitutive expression of HSPs is reduced in diabetics $[43,44]$. However, Najemnikova et al. [45] pointed out that diabetes alone is an insufficient stressor to alter the constitutive expression of HSPs in muscle tissue of STZ-induced diabetic rats. The reasons for these contradictory reports are numerous, including differences in the examined tissues, diabetic models, experimental duration, and severity of diabetes, as well as other factors.

Interestingly, we observed increased expression of muscular phosphoglucomutase-1 (PGM-1) in diabetic male and female rats. This glycolytic enzyme plays a pivotal role in glycogen metabolism by catalyzing the interconversion of glucose-1-phosphate and glucose6-phosphate and is reduced in type 1 muscle fibers [29]. Thus, elevated levels of PGM-1 in diabetic rat muscle indicate a reduced glycolytic-to-oxidative ratio in the utilization of energy mediated by insulin deficiency. In contrast, expression of $\beta$-enolase (Eno3) was reduced in both male and female diabetic rats. Although decreased glycolysis and increased gluconeogenesis are typical pathogenic features of diabetes, whole glycolytic enzymes are not likely to play pivotal roles in this event since they are multifunctional. Thus, altered expression of individual glycolytic enzymes does not directly affect whole muscle glycolysis, although it may play a specific role in muscle energetic and diabetic adaptation $[33,46]$.

Decreased expression of glycogen phosphorylase (GP) in diabetic male and female rats also drew our attention. Reports in the literature on the expression patterns of GP in diabetics are often contradictory. Earlier biochemical studies demonstrated that GP transcript levels are decreased in STZ-induced diabetic rat liver [47] and dystrophic mice muscle [48]. Conversely, elevated expression of the GP gene was observed in STZ-induced diabetic rat muscle [49]. Reduced levels of GP were likely the result of insulin deficiency in 
diabetic rats, as insulin plays a role in the synthesis of GP [47]. However, in comparison with the results of this study, GP inhibition was suggested as a possible therapy for T2DM since most patients with T2DM exhibit higher GP activity in most metabolic tissues [50]. Further studies are required to more definitely determine the expression of GP in the diabetic state.

We, for the first time, identified two proteins showing opposite regulation patterns between males and females. Valosin-containing protein (VCP) was up-regulated in diabetic male rats, whereas it was down-regulated in female diabetic rats compared to their healthy control littermates. VCP is known to participate in the degradation of misfolded ERsynthesized proteins, whereas its role in skeletal muscle is currently unknown [51]. We failed to interpret the opposite expression patterns of VCP between males and females, and thus the physiological significance of these changes remains to be determined. Conversely, alpha-enolase (Eno1) was down-regulated in male diabetic rats but up-regulated in female diabetic rats. This discrepant expression suggests the possibility that Eno1 may have other cellular functions distinct from its well-established function in glycolysis [52].

In conclusion, the results of our proteomic study indicate the existence of sex-associated differences in the regulation of skeletal muscle proteins between healthy control and STZ-induced diabetic rats. For the identified proteins showing gender-specific dimorphic regulation, further functional investigations are necessary to investigate how physiological levels of sex hormones or what other metabolic changes contribute to these differences. Overall, the current proteomic study found that impaired protein regulation was more severe in female diabetic rats, which were more susceptible to STZ-induced diabetes onset. We expect that the present proteomic data can provide valuable information for evidencebased gender-specific treatment of diabetes.

\section{Abbreviations}

2-ODGH (2-oxoglutarate dehydrogenase); AtpB (ATP synthase beta subunit); Eno1 (alpha-enolase); Eno3 (beta-enolase); G3pdh (glycerol-3-phosphate dehydrogenase); Gda (Guanine deaminase); GP (glycogen phosphorylase); Guk (guanylate kinase); HSP60 (heat shock protein 60); HSPB1 (heat shock protein beta-1); MHC (Myosin heavy chain); MYL6B (Myosin light chain 6B); MyBP-H (Myosin binding protein H); ND1 (NADH dehydrogenase subunit 1); PGM-1 (phosphoglucomutase-1); T1DM (type 1 diabetes mellitus); T2DM (type 2 diabetes mellitus); TCA (tricarboxylic acid); TPM1 (Tropomyosin alpha chain); TPM2 (Tropomyosin beta chain); SCoAL (succinyl-CoA ligase); SDH (succinate dehydrogenase); STZ (streptozotocin); VCP (valosin-containing protein).

\section{Conflict of interest}

The authors have declared no conflict of interest.

\section{Acknowledgements}

This work was supported by the SRC Program (Center for Food \& Nutritional Genomics: grant number 2012-0000638) of the National Research Foundation of Korea (NRF) funded by the Ministry of Education, Science and Technology.

\section{References}

1 LeRoith D: Gender differences in metabolic disorders. Gend Med 2009;6:S1-3.

Gale EA, Gillespie KM: Diabetes and gender. Diabetologia 2001;44:3-15. 


\begin{tabular}{|c|c|c|}
\hline Cello & Cell Physiol Biochem 2013;31:408-420 & \\
\hline and Biochemistry & $\begin{array}{l}\text { DOI: 10.1159/000343378 } \\
\text { Published online: March } 12,2013\end{array}$ & $\begin{array}{l}\text { O } 2013 \text { S. Karger AG, Basel } \\
\text { www.karger.com/cpb }\end{array}$ \\
\hline
\end{tabular}

3 Legato MJ, Gelzer A, Goland R, Ebner SA, Rajan S, Villagra V, Kosowski M: Gender-specific care of the patient with diabetes: Review and recommendations. Gend Med 2006;3:131-158.

4 Szalat A, Raz I: Gender-specific care of diabetes mellitus: Particular considerations in the management of diabetic women. Diabetes Obes Metab 2008;10:1135-1156.

5 Perreault L, Ma Y, Dagogo-Jack S, Horton E, Marrero D, Crandall J, Barrett-Connor E: Sex differences in diabetes risk and the effect of intensive lifestyle modification in the diabetes prevention program. Diabetes Care 2008;31:1416-1421.

6 Maahs DM, West NA, Lawrence JM, Mayer-Davis EJ: Epidemiology of type 1 diabetes. Endocrinol Metab Clin North Am 2010;39:481-497.

7 Rossini AA, Like AA, Chick WL, Appel MC, Cahill GF Jr: Studies of streptozotocin-induced insulitis and diabetes. Proc Natl Acad Sci U S A 1977;74:2485-2489.

$\checkmark 8$ Szkudelski T: The mechanism of alloxan and streptozotocin action in b cells of the rat pancreas. Physiol Res 2001;50:537-546.

9 Wei M, Ong L, Smith MT, Ross FB, Schmid K, Hoey AJ, Burstow D, Brown L: The streptozotocin-diabetic rat as a model of the chronic complications of human diabetes. Heart Lung Circ 2003;12:44-50.

10 Paik SG, Michelis MA, Kim YT, Shin S: Induction of insulin-dependent diabetes by streptozotocin. Inhibition by estrogens and potentiation by androgens. Diabetes 1982;31:724-729.

11 Le May C, Chu K, Hu M, Ortega CS, Simpson ER, Korach KS, Tsai MJ, Mauvais-Jarvis F: Estrogens protect pancreatic beta-cells from apoptosis and prevent insulin-deficient diabetes mellitus in mice. Proc Natl Acad Sci USA 2006;103:9232-9237.

$\rightarrow 12$ Vital P, Larrieta E, Hiriart M: Sexual dimorphism in insulin sensitivity and susceptibility to develop diabetes in rats. J Endocrinol 2006;190:425-432.

13 Macotela Y, Boucher J, Tran TT, Kahn CR: Sex and depot differences in adipocyte insulin sensitivity and glucose metabolism. Diabetes 2009;58:803-812.

14 Wild S, Roglic G, Green A, Sicree R, King H: Global prevalence of diabetes: estimates for the year 2000 and projections for 2030. Diabetes Care 2004;27:1047-1053.

15 Liu S, Mauvais-Jarvis F: Minireview: Estrogenic protection of beta-cell failure in metabolic diseases. Endocrinology 2010;151:859-864.

16 Ohlendieck K: Skeletal muscle proteomics: Current approaches, technical challenges and emerging techniques. Skelet Muscle 2011;1:6.

17 Gomez-Perez Y, Amengual-Cladera E, Catala-Niell A, Thomas-Moya E, Gianotti M, Proenza AM, Llado I: Gender dimorphism in high-fat-diet-induced insulin resistance in skeletal muscle of aged rats. Cell Physiol Biochem 2008;22:539-548.

18 Miller AE, MacDougall JD, Tarnopolsky MA, Sale DG: Gender differences in strength and muscle fiber characteristics. Eur J Appl Physiol Occup Physiol 1993;66:254-262.

19 Mierzejewska-Krzyzowska B, Drzymala-Celichowska H, Celichowski J: Gender differences in the morphometric properties of muscle fibres and the innervation ratio of motor units in rat medial gastrocnemius muscle. Anat Histol Embryol 2011;40:249-255.

$\checkmark 20$ Colom B, Alcolea MP, Valle A, Oliver J, Roca P, Garcia-Palmer FJ: Skeletal muscle of female rats exhibit higher mitochondrial mass and oxidative-phosphorylative capacities compared to males. Cell Physiol Biochem 2007;19:205-212.

-21 Catala-Niell A, Estrany ME, Proenza AM, Gianotti M, Llado I: Skeletal muscle and liver oxidative metabolism in response to a voluntary isocaloric intake of a high fat diet in male and female rats. Cell Physiol Biochem 2008;22:327-336.

22 Clark BC, Manini TM, The DJ, Doldo NA, Ploutz-Snyder LL: Gender differences in skeletal muscle fatigability are related to contraction type and emg spectral compression. J Appl Physiol 2003;94:2263-2272.

-23 Bradford MM: A rapid and sensitive method for the quantitation of microgram quantities of protein utilizing the principle of protein-dye binding. Anal Biochem 1976;72:248-254.

24 Kim DH, Choi JW, Joo JI, Wang X, Choi DK, Oh TS, Yun JW: Changes in expression of skeletal muscle proteins between obesity-prone and obesity-resistant rats induced by a high-fat diet. J Proteome Res 2011;10:12811292.

-25 Oh TS, Choi JW, Choi DK, Mukherjee R, Liu H, Yun JW: Gender dimorphism in skeletal muscle proteome between lean and diet-induced obese rats. Cell Physiol Biochem 2011;28:981-996.

26 Shevchenko A, Wilm M, Vorm 0, Mann M: Mass spectrometric sequencing of proteins silver-stained polyacrylamide gels. Anal Chem 1996;68:850-858.

27 Kim DH, Joo JI, Choi JW, Yun JW: Differential expression of skeletal muscle proteins in high-fat diet-fed rats in response to capsaicin feeding. Proteomics 2010;10:2870-2881. 
Choi et al.: Gender Dimorphism in Skeletal Muscle Proteome

28 Ordonez P, Moreno M, Alonso A, Fernandez R, Diaz F, Gonzalez C: Insulin sensitivity in streptozotocininduced diabetic rats treated with different doses of 17beta-oestradiol or progesterone. Exp Physiol 2007;92:241-249.

-29 Hojlund K, Wrzesinski K, Larsen PM, Fey SJ, Roepstorff P, Handberg A, Dela F, Vinten J, McCormack JG, Reynet C, Beck-Nielsen H: Proteome analysis reveals phosphorylation of atp synthase beta -subunit in human skeletal muscle and proteins with potential roles in type 2 diabetes. J Biol Chem 2003;278:1043610442.

-30 Jove M, Salla J, Planavila A, Cabrero A, Michalik L, Wahli W, Laguna JC, Vazquez-Carrera M: Impaired expression of nadh dehydrogenase subunit 1 and ppargamma coactivator-1 in skeletal muscle of zdf rats: Restoration by troglitazone. J Lipid Res 2004;45:113-123.

-31 Mullen E, Ohlendieck K: Proteomic analysis of the mitochondria-enriched fraction from diabetic rat skeletal muscle. JIOMICS 2011;1:108-114.

32 Large V, Beylot M: Modifications of citric acid cycle activity and gluconeogenesis in streptozotocin-induced diabetes and effects of metformin. Diabetes 1999;48:1251-1257.

33 Johnson DT, Harris RA, French S, Aponte A, Balaban RS: Proteomic changes associated with diabetes in the bb-dp rat. Am J Physiol Endocrinol Metab 2009;296:E422-432.

-34 Vandenburgh HH, Karlisch P, Shansky J, Feldstein R: Insulin and IGF-I induce pronounced hypertrophy of skeletal myofibers in tissue culture. Am J Physiol 1991;260:C475-484.

-35 Charlton MR, Balagopal P, Nair KS: Skeletal muscle myosin heavy chain synthesis in type 1 diabetes. Diabetes 1997;46:1336-1340.

-36 Perry SV: What is the role of tropomyosin in the regulation of muscle contraction? J Muscle Res Cell Motil 2004;24: 593-596.

-37 Tajsharghi H, Ohlsson M, Palm L, Oldfors A: Myopathies associated with beta-tropomyosin mutations. Neuromuscul Disord 2012;22:923-933.

-38 Hundley AF, Yuan L, Visco AG: Skeletal muscle heavy-chain polypeptide 3 and myosin binding protein $\mathrm{h}$ in the pubococcygeus muscle in patients with and without pelvic organ prolapse. Am J Obstet Gynecol 2006;194:1404-1410.

-39 Malhotra A, Sanghi V: Regulation of contractile proteins in diabetic heart. Cardiovasc Res 1997;34:34-40.

40 Yohannes E, Chang J, Christ GJ, Davies KP, Chance MR: Proteomics analysis identifies molecular targets related to diabetes mellitus-associated bladder dysfunction. Mol Cell Proteomics 2008;7:1270-1285.

41 Buccino RJ Jr, Roth JS: Partial purification and properties of atp: Gmp phosphransferase from rat liver. Arch Biochem Biophys 1969;132:49-61.

-42 Weber G, Lui MS, Jayaram HN, Pillwein K, Natsumeda Y, Faderan MA, Reardon MA: Regulation of purine and pyrimidine metabolism by insulin and by resistance to tiazofurin. Adv Enzyme Regul 1985;23:81-99.

43 Swiecki C, Stojadinovic A, Anderson J, Zhao A, Dawson H, Shea-Donohue T: Effect of hyperglycemia and nitric oxide synthase inhibition on heat tolerance and induction of heat shock protein $72 \mathrm{kda}$ in vivo. Am Surg 2003;69:587-592.

44 Atalay M, Oksala NK, Laaksonen DE, Khanna S, Nakao C, Lappalainen J, Roy S, Hanninen O, Sen CK: Exercise training modulates heat shock protein response in diabetic rats. J Appl Physiol 2004;97:605-611.

45 Najemnikova E, Rodgers CD, Locke M: Altered heat stress response following streptozotocin-induced diabetes. Cell Stress Chaperones 2007;12:342-352.

46 Ohlendieck K: Pathobiochemical changes in diabetic skeletal muscle as revealed by mass-spectrometrybased proteomics. J Nutr Metab 2012;2012:893-876.

47 Rao PV, Pugazhenthi S, Khandelwal RL: The effects of streptozotocin-induced diabetes and insulin supplementation on expression of the glycogen phosphorylase gene in rat liver. J Biol Chem 1995;270:24955-24960.

48 Leyland DM, Beynon RJ: The expression of glycogen phosphorylase in normal and dystrophic muscle. Biochem J 1991;278:113-117.

49 Reynet C, Kahn CR, Loeken MR: Expression of the gene encoding glycogen phosphorylase is elevated in diabetic rat skeletal muscle and is regulated by insulin and cyclic AMP. Diabetologia. 1996;39:183-189.

50 Baker DJ, Timmons JA, Greenhaff PL: Glycogen phosphorylase inhibition in type 2 diabetes therapy: A systematic evaluation of metabolic and functional effects in rat skeletal muscle. Diabetes 2005;54:24532459.

-51 Weihl CC, Pestronk A, Kimonis VE: Valosin-containing protein disease: Inclusion body myopathy with paget's disease of the bone and fronto-temporal dementia. Neuromuscul Disord 2009;19:308-315.

-52 Diaz-Ramos A, Roig-Borrellas A, Garcia-Melero A, Lopez-Alemany R: Alpha-enolase, a multifunctional protein: Its role on pathophysiological situations. J Biomed Biotechnol 2012;2012:156795. 